\section{P3-S3.06 OUANTIFYING LACTOBACILLAE SPECIES WITH REAL TIME PCR METHODS FOR HIV PREVENTION TRIALS}

doi:10.1136/sextrans-2011-050108.455

T Crucitti, V Jespers, J Menten, H Smet, A Buvé, S Poradosú. Institute of Tropical Medicine, Antwerpen, Belgium

Background The vaginal flora plays an important role in the defence against HIV infection. It is thus important to assess the impact on the vaginal milieu of products that are developed to protect women against HIV acquisition.

Objectives Using Real Time PCR, describe and quantify Lactobacillae spp. in healthy European women who enter microbicide phase I studies.

Methods Thirty healthy women who don't use hormones and have a regular menstrual cycle, were sampled with a COPAN flocked vaginal swab on days 7 and 21 of the cycle for a total of 5 visits. Real Time PCR was used to quantify bacterial cells for four Lactobacillus spp. at all visits.

Results $L$ crispatus, $L$ iners, $L$ jensenii, and $L$ gasseri were present at least once in $93 \%, 77 \%, 73 \%$ and $70 \%$ of women, respectively. High counts ( $\geq 8 \log$ for $L$ crispatus, $\geq 6 \log$ for other species) were consistently present in $60 \%, 67 \%, 53 \%$, and $53 \%$ of women. We labelled these latter women "L species-positive". Using logistic regression we modelled the probability of belonging to this group. The temporal variation of the log counts within this group was modelled using random effects linear regression. No predictors of being "L. species-positive" for any of the studied Lactobacillae were identified. Longitudinal analysis showed that $L$ crispatus counts were $0.22 \log$ higher $(p<0.001)$ and $L$ iners counts were $0.83 \log$ lower $(p<0.001)$ at the end of the menstrual cycle. Furthermore, $L$ crispatus counts decreased by $0.40 \mathrm{log}$ after intercourse (PSA present $)(p=0.002)$, while $L$ iners $(+0.73 \log , p=0.033)$ and $L$ gasseri $(+0.58$ log, $\mathrm{p}=0.052)$ increased see Abstract P3-S3.06 figure 1.

Conclusion The menstrual cycle and sexual activity have a significant effect on Lactobacillus sp. counts. This should be taken into account when assessing safety of products in HIV prevention trials.
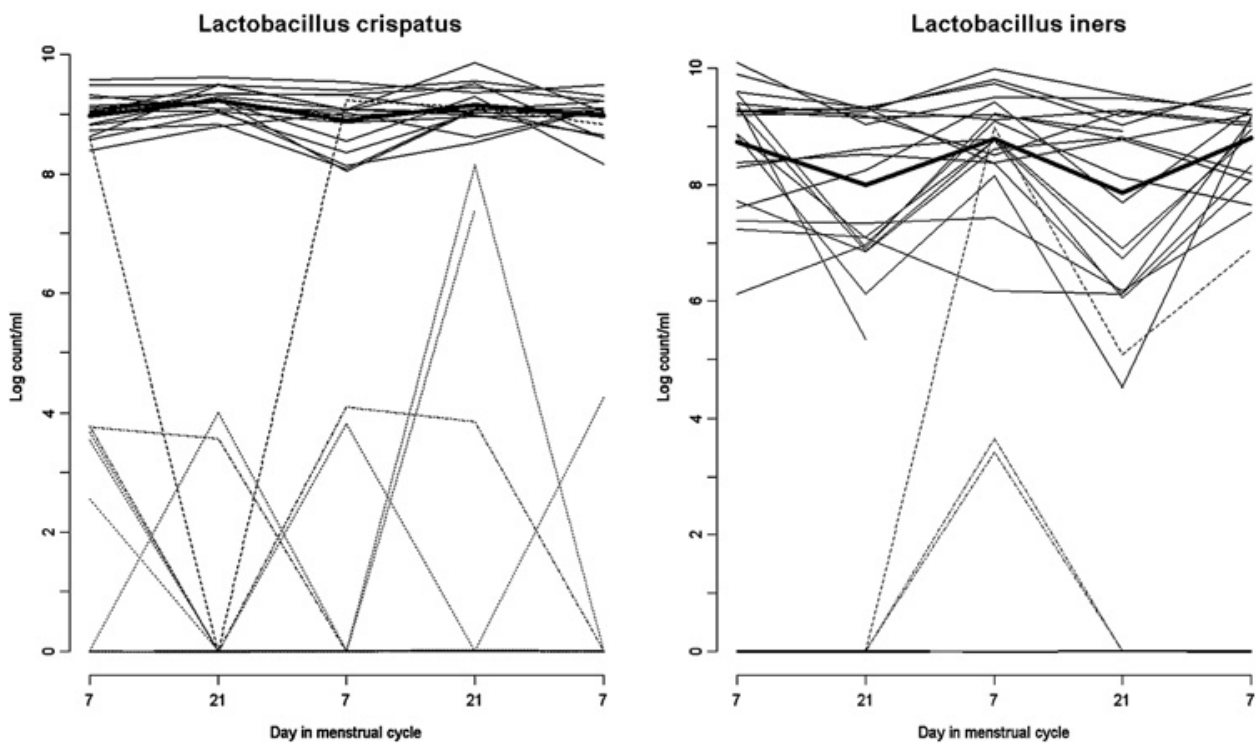

Abstract P3-S3.06 Figure 1 Log counts of bacterial cells per $\mathrm{ml}$ by day in the menstrual cycle: Note: Lines connect consecutive counts for individual women. Bold line is overall trend in "L. species-positive" women.

\section{P3-S3.07 ANALYSIS OF PATIENTS WHO DO NOT KEEP OR CANCEL APPOINTMENTS AT A UNIVERSITY BASED HIV/AIDS CLINIC}

doi:10.1136/sextrans-2011-050108.456

${ }^{1} \mathrm{~J}$ Edwards, ${ }^{2} \mathrm{R}$ C Parrish, ${ }^{3} \mathrm{~K}$ Frieson, ${ }^{2} \mathrm{~T}$ Crawford, ${ }^{4} \mathrm{~A}$ Thornton. ${ }^{1}$ University of Kentucky, Colleage of Public Health, Lexington, USA; ${ }^{2}$ University of Kentucky, Lexington, USA; ${ }^{3}$ University of Kentucky, Department of Educational and Counselling Psychology, Lexington, USA; ${ }^{4}$ University of Kentucky, College of Medicine, Lexington, USA

Background The Bluegrass Care Clinic (BCC), a university based HIV/AIDS clinic, has an annual average patient no show rate of $13.6 \%$ to medical appointments. Treatment adherence greater than $95 \%$ is needed to obtain maximum benefits of therapy and minimal disease progression requiring attendance to regularly scheduled medical appointments throughout the patient's lifespan. Missed appointments are not only known to interfere with appropriate care but to misspend medical and administrative resources. The BCC sought to identify demographic trends, interpret perception and identify barriers unique to those individuals who frequently do not attend appointments

Methods Quantitative and qualitative data were collected through focus groups, phone surveys and data from Resource Scheduling Software. Descriptive statistics (frequencies and means) were derived. Results During a 29 month period the BCC expended roughly $\$ 3864$ per month due to patients who did not show for appointments. 68 individual patients did not show for more than eight appointments. These 68 patients accounted for $21 \%$ of missed appointments, while 1053 patients accounted for the other $79 \%$ of missed appointments see Abstract P3-S3.07 figure 1. 13 patient focus groups were held where members cited transportation/coping/stress as potential barriers to attendance. During a phone survey of the 68 individuals only one individual correctly estimated the average number of appointments they missed per year. Female clients seem to disproportionately become frequent non-attenders as compared to their male counterparts. Female clients make up only $20 \%$ of the general BCC population, while they make up $34 \%$ of the clients who frequently miss appointments. There is a disproportionate effect in black clients. These individuals make up $21 \%$ of the BCC's total 